\title{
LEMNA MINOR L. AS A SOURCE OF ANTIOXIDANTS
}

\author{
V. Petrova-Tacheva ${ }^{*}$, V. Ivanov $^{2}$, A. Atanasov $^{3}$ \\ ${ }^{1}$ Department of Molecular Biology, Immunology and Medical Genetics, Faculty of Medicine, \\ Trakia University, Stara Zagora, Bulgaria \\ ${ }^{2}$ Department of Neurology, Psychiatry and MDS, Faculty of Medicine, Trakia University, \\ Stara Zagora, Bulgaria \\ ${ }^{3}$ Department of Physics and Biophysics, Faculty of Medicine, Trakia University, Stara Zagora, \\ Bulgaria
}

\begin{abstract}
Lemna Minor L. (LM) is a perennial aquatic plant that belongs to the genus Lemna, the family Lemnaceae and is spread almost ubiquitously. In folk medicine, it is used in the treatment of allergies, asthma, vitiligo, jaundice, glaucoma, rheumatism, gout, and others. In recent years it has become clear that its chemical composition consists of: proteins (up to 35\%), vegetable fibers (up to 17\%), fats (up to $5 \%$ ), polysaccharides, flavonoids, amino acids, aliphatic acids, phenolic acids, triterpene compounds, vitamins, micro- and macro-elements and other substances. A significant part of these substances exhibit the antioxidant activity. The scientific information for 12 biologically active substances with antioxidant activity isolated from Lemna Minor is presented in the review.
\end{abstract}

Key words: Lemna Minor, antioxidant activity

\section{INTRODUCTION}

Lemna Minor L. (LM) is a perennial aquatic plant that belongs to the genus Lemna, the family Lemnaceae and is spread almost ubiquitously. In folk medicine it is used in the treatment of allergies, asthma, vitiligo, jaundice, glaucoma, rheumatism, gout and others (1). In recent years it has become clear that its chemical composition consists of: proteins (up to $35 \%$ ), vegetable fibers (up to $17 \%$ ), fats (up to 5\%), polysaccharides, flavonoids, amino acids, aliphatic acids, phenolic acids, triterpene compounds, vitamins, micro- and macro-elements and other substances (2-6). Vladimirova and Georgiyants, investigating LM, identify the presence of 32 biologically active substances from different chemical groups. The largest quantities represented were those of the phytosterols $\quad(52.8 \quad \mathrm{mg} / \mathrm{kg}), \quad$ saturated hydrocarbons $(23.1 \mathrm{mg} / \mathrm{kg})$, aldehydes and

\footnotetext{
*Correspondence to: Veselina Petrova-Tacheva, Department of Molecular Biology, Immunology and Medical Genetics, Faculty of Medicine, Trakia University, Armeiska 11, 6000 Stara Zagora, Bulgaria Tel.:042/664311, Mobile: 0896056412, email:vesepetr@abv.bg
}

ketones $(20.2 \mathrm{mg} / \mathrm{kg})$, fatty acids and their derivatives (11.1 mg/kg) (6). Gulcin et al., through a series of in vitro tests, found out that aqueous and ethanol extracts of LM have antioxidant and antiradical effects (7). Later, another research group discovered and described that the methanol extract from LM exhibits significant antioxidant activity (8). Although data on the phytochemical composition of LM and its extracts are increasing, and their antioxidant and antiradical properties have already been established, questions about the mechanisms of their antioxidant activity not yet been fully elucidated, as well as to which of their ingredients these are due. For this reason, in our study, we set before ourselves the aim to find and describe, on the basis of the available scientific literature, the compounds with proven antioxidant activity, which have so far been isolated from the Lemna Minor L. plant and its extracts, respectively.

\section{MATERIALS AND METHODS}

For each of the so far identified compounds isolated from the plant Lemna Minor L. and from its extracts, a survey was conducted for the presence of antioxidant properties. The 
main electronic sources of information we used, are PubMed, Google Scholar and eLIBRARY.RU. The search was conducted using the combination of keywords obligatorily including the chemical name of the compound (including synonyms) and the words antioxidant, antiradical and oxygen free radicals in Bulgarian, English, and Russian.

\section{RESULTS AND DISCUSSION}

As a result of the study, we found out 12 substances with antioxidant activity isolated from the plant Lemna Minor L. These compounds are: phytol, campesterol, loliolide, dihydroactinidiolide, ascorbic acid, vanillic acid, 2,3-dihydroxybenzoic acid, caffeic acid, chlorogenic acid, esculetin, esculin and fraxetin.

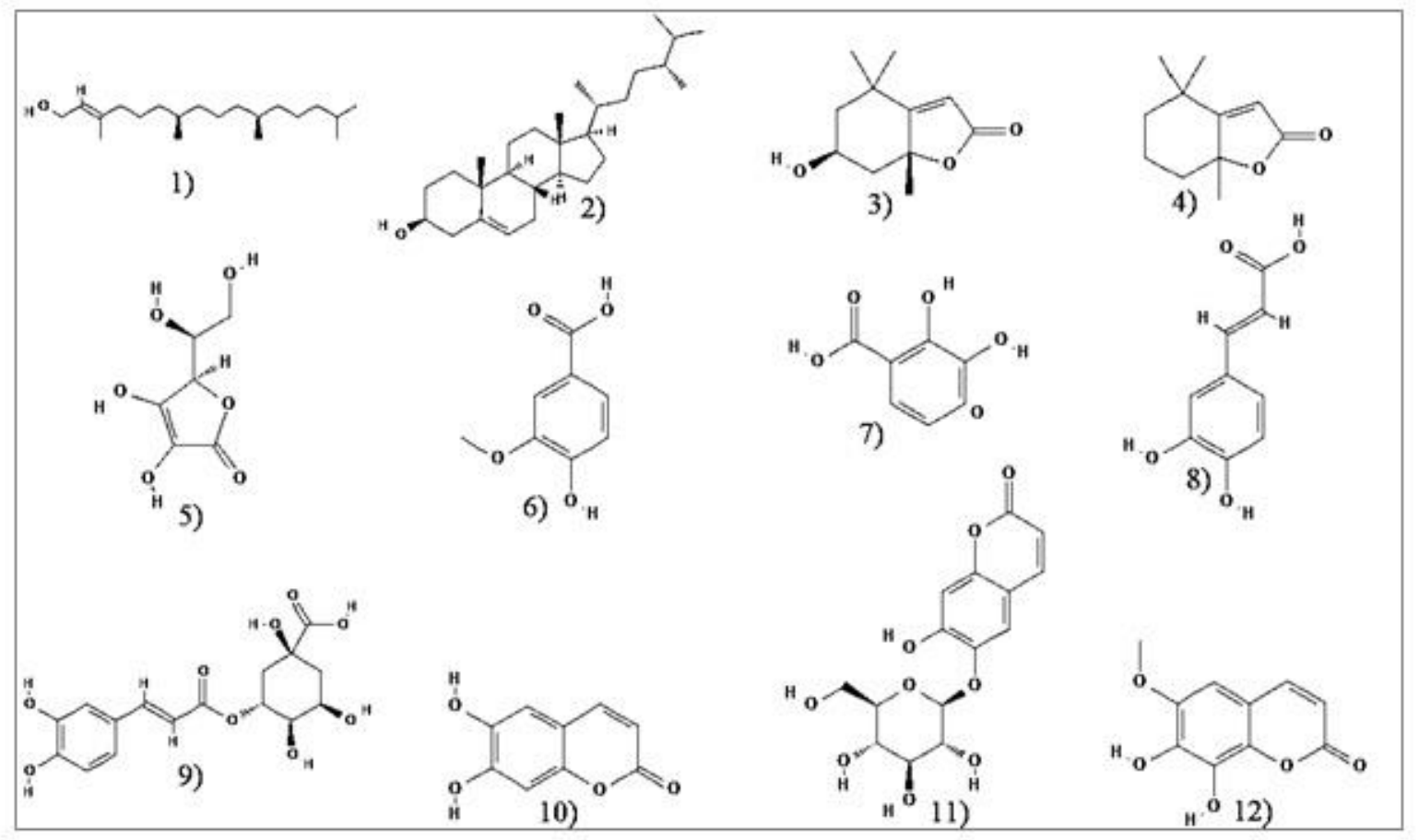

Figure 1.2D molecular structures of 12 biological substances with antioxidant activity, isolated from the plant Lemna Minor L.: 1) phytol; 2) campesterol; 3) loliolide; 4) dihydroactinidiolide; 5) ascorbic acid; 6) vanillic acid; 7) 2,3-dihydroxybenzoic acid; 8) caffeic acid; 9) chlorogenic acid; 10) esculetin;11) esculin; 12) fraxetin. The molecular structures are taken from: https://pubchem.ncbi.nlm.nih.gov.

\section{Phytol}

Phytol (3, 7, 11, 15-tetramethylhexadec-2-en$1-\mathrm{ol})$ is a diterpene and is a member of the group of branched-chain unsaturated alcohols (Figure 1.1) (9). Its measured amount in the dry substance from Lemna Minor is $3.6 \mathrm{mg} / \mathrm{kg}$ (6). To this date, a number of in vitro, ex vivo and in vivo test systems have been described in the scientific literature, demonstrating its potent antioxidant and antiradical properties $(9$, 10-12). The in vitro tests have shown that it removes the formed hydroxyl radicals and nitric oxide and prevents the formation of thiobarbituric acid reactive substances (9). Investigating its antiradical activity through electron paramagnetic resonance, it was found out that it reduces the production of all the tested radicals (hydroxyl radical, superoxide anion radical, methoxy radical, carbon dioxide anion radical, nitric oxide radical and 2,2diphenyl-1-picrylhydrazyl radical), with greater activity against carbon dioxide anion radical, methoxy radical and 2,2-diphenyl-1picrylhydrazyl radicals (10). In an in vivo study with a Saccharomyces cerevisiae test, the pretreatment with phytol protects against $\mathrm{H}_{2} \mathrm{O}_{2}$-induced damages, in the co-treatment group the phytol exhibits significant antioxidant activity, and in the post-treatment group its application it enhances significantly the reparative processes (11). In experiments with Swiss mice its intraperitoneal application results in a decrease in lipid peroxidation and nitrite contents in the homogenates obtained 
from mice hippocampi and in an increase in the reduced glutathione, increasing of the activity of the superoxide dismutase and the catalase (11). In another experiment with Wistar rats, the administration of phytol protects the kidney tissue against the damage caused by the oxidative stress and inflammation caused by hyperglycemia in diabetes mellitus (12).

\section{Campesterol}

Campesterol (Figure 1.2) is one of the most common plant sterols in nature, along with $\beta$ sitosterol and stigmasterol. It is found in seeds, nuts, cereals, beans, legumes, and vegetable oils (13). Its amount in the dry substance from Lemna Minor is $7.2 \mathrm{mg} / \mathrm{kg}$ (6). Campesterol has been shown to have an antioxidant effect on the oxidation of methyl linoleate in solution and its effect is similar to that of the phytosterol and $\beta$-sitosterol (14).

\section{Loliolide}

Loliolide (Figure 1.3) is a member of benzofurans (15). The amount of loliolide in the dry substance from Lemna Minor is 2.3 $\mathrm{mg} / \mathrm{kg}$ (6). Through a series of in vitro experiments, loliolide was found to exhibit moderate antioxidant activities in all antioxidant studies (DPPH and $\mathrm{H}_{2} \mathrm{O}_{2}$ radical and intracellular reactive oxygen species scavenging assays)(16).

\section{Dihydroactinidiolide}

Dihydroactinidiolide (Figure 1.4) is isolated from tea, coffee, and fruits. It is an important aroma constituent of tea (17). The amount of dihydroactinidiolide in dry matter from Lemna Minor is $2.0 \mathrm{mg} / \mathrm{kg}$ (6). It has been found to exhibit antioxidant activity, antibacterial activity, anticancer activity and neuroprotective effect $(18,19)$.

\section{Ascorbic acid}

Ascorbic acid (AA, vitamin C) is a watersoluble ketolactone with two ionizable hydroxyl groups (20) (Figure 1.5). The quantity of the ascorbic acid is $23.55 \pm 2.29$ $\mathrm{nmol} / \mathrm{g}$ fresh weight of Lemna Minor plants grown on Pirson-Seidel nutrient medium (21). Its antioxidant properties have been well documented and described. It is a powerful reducing agent and scavenger of free radicals in biological systems. It works effectively both inside and outside the cells and can neutralize free radicals and thus prevent free radical damage. It participates in the first line of antioxidant protection, protecting lipid membranes and proteins from oxidative damage. Vitamin $\mathrm{C}$ can donate electrons to free radicals and quench their reactivity. It has been found that vitamin $\mathrm{C}$ is an effective scavenger against oxygen and nitrogen oxide species, superoxide radical ion, hydrogen peroxide, hydroxyl radical, and singlet oxygen. It has also been found to be a cofactor for enzymes involved in the regulation of photosynthesis, hormone biosynthesis and the regeneration of other antioxidants. However, in the presence of redox-active ions (iron, copper), vitamin $\mathrm{C}$ may act as a pro-oxidant contributing to the formation of hydroxyl radicals that can trigger dangerous radical reactions $(22,23)$. The occurrence of ascorbic acid of anti- or pro-oxidant properties depends on the substrate concentration and conditions under which the oxidation reaction takes place (23).

\section{Vanillic acid}

Vanillic acid (4-hydroxy-3-methoxybenzoic acid, Figure 1.6), an oxidized form of vanillin, is a phenolic derivative of edible plants and fruits used as a flavoring agent $(24,25)$. The quantity of the vanillic acid is $0.03 \pm 0.002$ in polyphenolic complex derived from $70 \%$ alcoholic extract of Lemna Minor, recalculated according to the mass of the polyphenolic complex (26). Vanillic acid has a significant and powerful antioxidant effect, which has been described in numerous studies conducted in vitro and in vivo $(24,25,27-31)$.

\section{2,3-Dihydroxybenzoic acid}

2,3-dihydroxybenzoic acid (Figure 1.7) is a dihydroxybenzoic acid that is benzoic acid substituted by hydroxy groups at positions 2 and 3 (32). The amount of the compound is $0.008 \pm 0.0005$ in the polyphenolic complex derived from $70 \%$ alcoholic extract of Lemna Minor, recalculated according to the mass of the polyphenolic complex (26). In vitro experiments, examining the antioxidant properties of several iron chelators by five different tests investigating antioxidant properties, namely: the potassium ferricyanide reduction method, 1,1-diphenyl-2picrylhydrazyl radical scavenging activity, $\mathrm{H}_{2} \mathrm{O}_{2}$ scavenging activity and hydroxyl radical scavenging activity, it has been found out that 2,3-dihydroxybenzoic acid exhibits significant antioxidant properties (33). 
Caffeic acid

Caffeic acid (3,4-dihydroxycinnamic acid, (Figure 1.8) is a hydroxycinnamic acid derivative and polyphenol, with potential antioxidant, anti-inflammatory, and antineoplastic activities. Upon administration, it acts as an antioxidant and prevents oxidative stress, thereby preventing DNA damage induced by free radicals $(33,34)$. It is described that it can react with free radicals more effectively than Trolox $\AA$, a water-soluble vitamin $\mathrm{E}$ analog (35). The quantity of the caffeic acid is $0.030 \pm$ 0.002 in polyphenolic complex derived from $70 \%$ alcoholic extract of Lemna Minor, recalculated according to the mass of the polyphenolic complex (26).

\section{Chlorogenic acid}

Chlorogenic acid (Figure 1.9) is a caffeic acid ester and one of the stereoisomers of the quinic acid. It is widespread in nature, the largest amount being found in coffee beans, sunflower seeds, the leaves of bilberries and white poplar leaves, as well as chicory roots (36). The quantity of the chlorogenic acid is $0.27 \pm 0.02$ in polyphenolic complex derived from $70 \%$ alcoholic extract of Lemna Minor, recalculated according to the mass of the polyphenolic complex (26). Chlorogenic acid has strong antioxidant, antiviral, antibacterial, hypoglycemic, hypocholesterolemic, anticancer and hepatoprotective properties (36).

\section{Esculetin}

Esculetin (Figure 1.10) is a hydroxycoumarin that is umbelliferone in which the hydrogen at position 6 is substituted by a hydroxy group (37). The quantity of esculetin in the polyphenolic complex derived from $70 \%$ alcoholic extract of Lemna Minor, recalculated according to the mass of the polyphenolic complex, is $0.46 \pm 0.04$ (36). Esculetin is a proven antioxidant (38) with high antioxidant activity, compared to other tested coumarins (39). Esculetin exhibits strong scavenging activity against DPPH radicals, which shows a significant linear correlation both in terms of time and concentration. Esculetin is also a potent agent for protecting cells from oxidative stress caused by lipid peroxidation, protein carbonyl, and DNA damages (39-42).

\section{Esculin}

Esculin (Figure 1.11) is a hydroxycoumarin that is the 6-O-beta-D-glucoside of esculetin (43). It also exhibits antioxidant, antibacterial, anti-inflammatory, antihistamine, vitamin $\mathrm{P}$ like, and anti-carcinogenic effects (43-45). The quantity of the compound in the polyphenolic complex derived from $70 \%$ alcohol extract of Lemna Minor, recalculated according to the mass of polyphenolic complex, is $1.31 \pm 0.07$ (26).

\section{Fraxetin}

Fraxetin (Figure 1.12) is a hydroxycoumarin that is 6-methoxycoumarin in which the hydrogens at positions 7 and 8 have been replaced by hydroxy groups (46). The quantity of fraxetine in the polyphenolic complex derived from $70 \%$ alcoholic extract of Lemna Minor, recalculated according to the mass of the polyphenolic complex is $0.21 \pm 0.02$ (26). Its great antioxidant capacity has been studied in in vitro and in vivo experiments $(46,47)$.

\section{CONCLUSIONS}

The substances described above can explain the antioxidant and antiradical effects of plant extracts from the Lemna Minor plant. In addition to the antioxidant and anti-radical properties described above, these substances also have many other useful and valuable pharmacological properties that may serve as a guide for future studies with LM extracts.

\section{ACKNOWLEDGMENT}

The studies were performed under scientific research projects №7/2017 and №14/2017, Trakia University, Stara Zagora, Bulgaria.

\section{REFERENCES}

1. Petrova-Tacheva, V., Alekova, S. and Ivanov, V., Lemna Minor L. and folk medicine. Science \& Technologies, 8(1):4246, 2018.

2. Kolomiets, N., Tueva, I., Mal'tseva, O., Dmitruk, B and Kalinkina, G., Estimation of the prospects of some types of herbal medicinal raw materials from the point of view of their ecological purity. Khimiya Rastit. Syr'ya, 4:25-28, 2004.

3. Nikiforov, L., Study of antifungal activity, sorption properties and bioelement compositions of Lemna minor and Lemna trisulca. Medicine in Kuzbass, 7:59-60, 2009.

4. Nikolaichuk, L., Zhigar, M., Healingplants. Medicinal properties. Culinary recipes. Application in cosmetics. Prapor, Kharkiv, Ukraine, 1992.

5. Soloveva, V., Folk methods for strengthening of the health. Neva, Saint Petersburg, Russia 2005. 
6. Vladimirova, I. and Georgiyants, V., Biologically Active Compounds from Lemna Minor S.

7. F. Grey. Pharmaceutical Chemistry Journal, 47(11):599-601, 2014.

8. Gulcin, I., Kirecci, E., Akkemik, E., Topal, F. and Hisar, H., Antioxidant and antimicrobial activities of an aquatic plant: Duckweed (Lemna minor L.). Turk J Biol, 34:175-188, 2010.

9. Bright, R. and Kanagappan, M., In vitro antioxidant activity of selected aquatic weeds of Kanyakumari district of south India. World Journal of Pharmacy and Pharmaceutical Sciences, 5(6):1090-1108, 2016.

10.Santos, C., Salvadori, M., Mota, V., Costa, L., Almeida, A., Oliveira, G., Costa, J., Sousa, D., Freitas, R. and Almeida, R., Antinociceptive and Antioxidant Activities of Phytol In Vivo and In Vitro Models. Neurosci J, 2013:1-9, 2013.

11.Pejin, B., Savic, A., Sokovic, M., Glamoclija, J., Ciric, A, Nikolic, M., Radotic, K. and Mojovic, M., Further in Vitro Evaluation of Antiradical and Antimicrobial Activities of Phytol. Nat Prod Res., 28(6):372-6, 2014.

12. Costa, J., Islam, M., Santos, P., Ferreira, P., Oliveira, G., Alencar, M., Paz, M., Ferreira, E., Feitosa, C., Citó, A., Sousa, D. and Melo-Cavalcante, A., Evaluation of Antioxidant Activity of Phytol Using NonAnd Pre-Clinical Models. Curr Pharm Biotechnol, 17(14):1278-1284, 2016.

13. Adeyomoye, O. and Adewoye, E., Phytol Reduces Oxidative Stress and Cyclooxygenase-2 Expression in Kidney of Diabetic Wistar Rats. Recent Adv Biol Med., 4:16-20, 2018.

14.Choi, J., Lee, E., Lee, H., Kim, K., Ahn, K., Shim, B., Kim, N., Song, M., Baek, N. and Kim, S., Identification of Campesterol From Chrysanthemum Coronarium L. And Its Antiangiogenic Activities. Phytother Res., 21(10):954-9, 2007.

15. Yoshida, Y. and Niki, E., Antioxidant Effects of Phytosterol and Its Components. J Nutr Sci Vitaminol, 49(4):277-80, 2003.

16.https://pubchem.ncbi.nlm.nih.gov/compoun $\mathrm{d} /$ loliolide

17.Yang, X., Kang, M., Lee, K., Kang, S., Lee, W. and J. Jeon, Y., Antioxidant activity and cell protective effect of loliolide isolated from Sargassum ringgoldianum subsp. coreanum. Algae, 26(2):201-208, 2011. 18.https://pubchem.ncbi.nlm.nih.gov/compoun d/Dihydroactinidiolide

19.https://www.medchemexpress.com/dihydro actinidiolide.html

20.Das, M., Prakash, S, Nayak, C., Thangavel, N., Singh,S., Manisankar, P. and Devi, K, Dihydroactinidiolide, a Natural Product Against $A \beta{ }_{25-35}$ Induced Toxicity in Neuro2a Cells: Synthesis, in Silico and in Vitro Studies. Bioorg Chem., 81:340-349, 2018.

21.Du, J., Cullen, J. and Buettner, G., Ascorbic Acid: Chemistry, Biology and the Treatment of Cancer. Biochim Biophys Acta, 1826(2):443-57, 2012.

22.Varga, M., Horvatic, J. and Celic, A., Short term exposure of Lemna minor and Lemna gibba to mercury, cadmium and chromium. Cent. Eur. J. Biol., 8(11):1083-1093, 2013.

23.Pehlivan, F., Vitamin C: An Antioxidant Agent. In: Vitamin C, A. Hamza (eds.), In Tech Open, 2017.

24.Mentschikova, E., Lankin, V., Zenkov, N., Bondar, I., Krugowih, N., Trufakin, V., Oxidative stress. Prooxidants and antioxidants. Slovo press, Moscow, Russia, 2006.

25.Kumar, S., Prahalathan, P. and Raja, B., Antihypertensive and antioxidant potential of vanillic acid, a phenolic compound in LNAME-induced hypertensive rats: a dosedependence study. Redox Rep., 16(5):208215, 2011.

26. Vinothiya, K. and Ashokkumar, N., Modulatory Effect of Vanillic Acid on Antioxidant Status in High Fat DietInduced Changes in Diabetic Hypertensive Rats. Biomed Pharmacother., 87:640-652, 2017.

27.Adekenov, S., Danilets, M., Ivasenko, S., Nikiforov, L., Krivoshchekov, S., Ligacheva, A., Trofimova, E., Sherstoboev, E., Zhdanov, V. and Belousov, M., Phenolic compounds of ethanol extracts of Lemna minor L., Lemna trisulca L. and Lemna polyrrhiza L. Schleid and their immunomodulating activity. Bulletin of Siberian Medicine, 16(3):5-15, 2017.

28.Zhao, D., Islam, M., Ahn, B., Jung, H., Kim, B. and Choi, J., In Vitro Antioxidant and Anti-Inflammatory Activities of Angelica Decursiva. Arch Pharm Res., 35(1):179-92, 2012.

29.Taner, G., Vardar, D., Aydin, S., Aytaç, Z., Başaran, A. and Başaran, N. Use of in vitro assays to assess the potential cytotoxic, genotoxic and antigenotoxic effects of 
vanillic and cinnamic acid. Drug Chem Toxicol., 40(2):183-190, 2017.

30.Chou, T., Ding, H., Hung, W. and Liang, C., Antioxidative Characteristics and Inhibition of Alpha-MelanocyteStimulating Hormone-Stimulated Melanogenesis of Vanillin and Vanillic Acid From Origanum Vulgare. Exp Dermatol., 19(8):742-50, 2010.

31.Amin, F., Shah, S. and Kim, M., Vanillic Acid Attenuates $A \beta$ 1-42-induced Oxidative Stress and Cognitive Impairment in Mice. Sci Rep., 7:40753, 2017.

32.Stanely Mainzen Prince, P., Rajakumar, S. and Dhanasekar, K., Protective Effects of Vanillic Acid on Electrocardiogram, Lipid Peroxidation, Antioxidants, Proinflammatory Markers and Histopathology in Isoproterenol Induced Cardiotoxic Rats. Eur J Pharmacol., 668(12):233-40, 2011.

33.https://pubchem.ncbi.nlm.nih.gov/compoun d/2_3-Dihydroxybenzoic-acid

34.Adjimani, J. and Asare, P., Antioxidant and Free Radical Scavenging Activity of Iron Chelators. Toxicol Rep., 2:721-728, 2015.

35.https://ncit.nci.nih.gov/ncitbrowser/Concept Report.jsp?dictionary=NCI_Thesaurus\&ns $=$ NCI_Thesaurus\&code $=\mathrm{C} 68540$

36.Kfoury, M., Geagea, C., Ruellan, S., Greige-Gerges, H. and Fourmentin, S., Effect of Cyclodextrin and Cosolvent on the Solubility and Antioxidant Activity of Caffeic Acid. Food Chem., 278:163-169, 2019.

37.Levitsky, A., Vertikova, O. and Selivansky, I., Chlorogenic acid: biochemistry and physiology. Microbiology and biotechnology, 2:6-20, 2010.

38.https://pubchem.ncbi.nlm.nih.gov/compoun d/Esculetin

39.Subramaniam, S. and Ellis, E., Esculetininduced protection of human hepatoma HepG2 cells against hydrogen peroxide is associated with the Nrf2-dependent induction of the $\mathrm{NAD}(\mathrm{P}) \mathrm{H}$ : Quinone oxidoreductase 1 gene. Toxicol Appl Pharmacol., 250(2): 130-136, 2011.
40.Witaicenis, A., Luchini, A., Hiruma-Lima, C., Felisbino, S., Justulin, L., Jr, GarridoMesa, N., Utrilla, P., Gálvez, J. and Di Stasi, L., Mechanism and Effect of Esculetin in an Experimental Animal Model of Inflammatory Bowel Disease. European Journal of Inflammation, 11(2):433-446, 2013.

41.Liang, C., Ju, W., Pei, S., Tang, Y. and Xiao, Y., Pharmacological Activities and Synthesis of Esculetin and Its Derivatives: A Mini-Review. Molecules, 22(3):387, 2017.

42.Lin, H., Tsai, S., Chen, C., Chang, Y., Lee, C., Lai, Z. and Lin, C., Structure-activity Relationship of Coumarin Derivatives on Xanthine Oxidase-Inhibiting and Free Radical-Scavenging Activities. Biochem Pharmacol., 75(6):1416-25, 2008.

43.Kim, S., Kang, K., Zhang, R., Piao, M., Ko, D., Wang, Z., Chae, S., Kang, S., Lee, K., Kang, H., Kang, H. and Hyun, J., Protective Effect of Esculetin Against Oxidative Stress-Induced Cell Damage via Scavenging Reactive Oxygen Species. Acta Pharmacol Sin., 29(11):1319-26, 2008.

44.https://pubchem.ncbi.nlm.nih.gov/compoun $\mathrm{d} /$ Esculin

45.Biljali, S., Hadjimitova, V., TopashkaAncheva, M., Momekova, D., Traykov, T. and Karaivanova, M., Antioxidant and antiradical properties of esculin, and its effect in a model of epirubicin-induced bone marrow toxicity. Folia Medica, 54(3):42-49, 2012.

46.Kang, K., Lee, W., Jung, Y., Lee, J., Lee, S., Eom, D., Jeon, Y., Yoo, H., Jin, M., Song, K., Kim, W., Ham, J., Kim, H. and Kim, S., Protective Effect of Esculin on Streptozotocin-Induced Diabetic Renal Damage in Mice, J Agric Food Chem., 62(9):2069-76, 2014. 